\title{
SAR IMAGE SIMULATION FOR THE ASSESSMENT OF DESPECKLING TECHNIQUES
}

\author{
Gerardo Di Martino, Mariana Poderico, Giovanni Poggi, Daniele Riccio, Luisa Verdoliva \\ Università di Napoli Federico II, Via Claudio 21, 80125, Napoli, Italy \\ \{gerardo.dimartino,mariana.poderico,poggi,daniele.riccio,verdoliv\}@unina.it
}

\begin{abstract}
We propose a new framework for the quantitative assessment of SAR despeckling techniques, based on physical-level simulation of SAR images corresponding to canonical scenes. Thanks to the simulator, we can generate multiple SAR images of the same scene which differ only in the speckle content, and, hence, a true multilook SAR image, with an arbitrarily large number of looks, to use as "speckle-free" reference. Based on this concept, we select a small set of canonical scenes and, for each of them, a suitable set of objective measures which account for speckle suppression and image feature preservation. We gain insight into the system reliability by comparing the indications it gives for some sample despeckling filters with those obtained by expert visual inspection of the filtered images.
\end{abstract}

Index Terms - Synthetic aperture radar (SAR), speckle reduction, quality assessment.

\section{INTRODUCTION}

With the increasing availability of very high resolution Synthetic Aperture Radar (SAR) data on a worldwide scale, the potential of SAR Earth Observation is experiencing a significant leap forward. However, exploiting this potential requires massive elaboration capabilities which cannot be granted by SAR experts only. Consequently, this huge amount of data calls for the development of automatic analysis tools in order to obtain value added products which can be more easily interpreted also by non-expert users [1]. Devising such tools is particularly challenging for SAR images, due to the inherent presence of speckle noise which tends to reduce their information extraction ability. Since a resolution cell is usually larger than the wavelength of the incident electromagnetic field, contributions coming from a single resolution cell sum up coherently, giving rise to interference phenomena that originate the speckle. Due to the lack of deterministic knowledge of the structure of the observed surface at the wavelength scale, a statistical description of SAR images is usually considered [2].

For many applications, speckle removal is fundamental in order to allow a better interpretation of SAR images. In fact, in the last decades, despeckling has been a very active research field and, nowadays, a significant number of new techniques is proposed each year in the remote sensing and signal processing communities. However, an objective performance comparison among such techniques is impossible for the lack of an established assessment protocol. Typically, authors of a new despeckling algorithm carry out some kind of comparison with the literature, but on proprietary images (barring reproducibility of results) and with ad hoc methodology. On the other hand, even the definition of performance is not obvious in this field, because of the lack of speckle-free reference images [3]. Under this point of view, SAR despeckling is quite unlike optical image denoising. In the latter field, one can use a virtually noise-free image as clean reference, add noise with the desired statistics, and measure performance by comparing the denoised image with the reference. In SAR no such thing as a speckle-free image is available because speckle is inherent of the imaging mechanism. Nonetheless, this assessment procedure is often applied also to SAR despeckling, using an optical image as clean reference, but the source image statistics will be obviously much different from those of a true SAR image, and the superimposed speckle noise will not be filtered according to the SAR system impulse response [4]. All in all, this approach does not provide reliable indications, and can be justified only by the lack of alternatives.

In this paper we try to overcome this problem by generating simulated reference images that can be regarded as legitimate speckle-free SAR images. To this end we resort to a SAR simulator, developed and successfully tested in the past two decades [5], in order to obtain realistic speckled SAR images. The simulator allows us to generate an arbitrarily large number of realizations of the same SAR image: hence, starting from this stack of images, we are able to perform a true statistical multilook obtaining as output an image presenting the desired, possibly very large, number of looks. Since a truly statistical multilook is carried out (i.e., without resorting to spatial averaging), the resulting image is not subject to any decrease in resolution and can be used effectively as clean reference for performance assessment. Given this general framework, the problem shifts to the selection of diagnostically significant test SAR images and to the definition of suitable performance measures that take into account the particular characteristics of interest in the considered image to measure both the speckle suppression power and the preservation of useful image features. 
The paper is organized as follows. In Section 2 we briefly describe the simulation framework and the generation of the speckle-free reference image. In Section 3 we show some meaningful examples of the obtained results and of the used performance measures. Finally, in Section 4 some concluding remarks are provided.

\section{SIMULATION FRAMEWORK}

The considered simulation framework allows the generation of a wide set of canonical SAR images, which can be used as a test-bed in order to use objective quality measures and assess the performance of the despeckling techniques. The proposed simulator is based on sound physical (geometric and electromagnetic) models [6], allowing the evaluation of the reflectivity function of the scene, and on a model for the transfer function of the system, which is used for the evaluation of the SAR raw signal [5]. It requires as input a Digital Elevation Model (DEM), a synthetic description of the roughness of the resolution cell, the electromagnetic parameters of the surface and the radar and orbital data of the sensor.

The simulation procedure allows the definition of speckle-free images. Considering that speckle is an intrinsic characteristic of SAR data, the notion of a noiseless SAR image is rather elusive if not plain contradictory. Nonetheless, if we look for an image whose macroscopic features are not affected by the presence of speckle, this coincides with a SAR image with an infinite number of looks, which we can call, with some abuse, noise-free. Of course, with real-world SAR systems, a true multilook, based on a large set of independent images of the same scene taken in the very same conditions, is all but impossible to obtain, and in fact practical multilook procedures refer to some kind of spatial averages, which reduce speckle at the cost of a decrease in the spatial resolution. However, thanks to the use of a simulator, we can obtain a SAR image presenting a finite but very large number of looks. In fact, it is possible to compute it as the average of a large number of independent images relevant to the same input DEM. In particular, in this paper the specklefree reference images are obtained by averaging 512 independent intensity images.

Finally, we note that the presented physical simulation allows generating SAR images and corresponding specklefree counterparts for any choice of the scene (dielectric constants, dimensions of the objects, contrast between objects and background) and sensor (wavelength, resolution, operational mode) parameters, thus providing the possibility to arrange specific benchmarks for particular despeckling frameworks.

\section{SAMPLE RESULTS}

In the following we show some meaningful examples of performance assessment on a very limited set of test cases
TABLE I

MEASURES FOR HOMOGENEOUS

\begin{tabular}{crrrr}
\hline \hline & MoI & MoR & ENL & DG \\
\hline Clean & 1.000 & 1.003 & 445.77 & \\
Noisy & 1.003 & & 0.99 & 0 \\
Frost & 1.002 & 0.676 & 8.12 & 9.12 \\
SAWBMMAE & 1.016 & 0.927 & 32.42 & 14.85 \\
SAR-BM3D & 0.985 & 0.813 & 90.69 & 19.16 \\
\hline \hline
\end{tabular}

and for just a few despeckling techniques. A deeper experimental analysis is presented in [7] together with a more thorough description of the proposed method. Here, we consider three test images: the first one ("Homogeneous") corresponds to a flat region with constant electromagnetic parameters; the second one ("DEM") to a non-flat scene, again with constant electromagnetic parameters; finally, the last case ("Corner") corresponds to a corner reflector placed in the middle of a flat area with constant electromagnetic parameters. Moreover, we consider three despeckling techniques, following the major SAR denoising approaches (linear filtering, wavelet shrinkage and nonlocal filtering, respectively), namely Frost filter [8], SA-WBMMAE [9] and SAR-BM3D [10].

\subsection{Homogeneous}

In Fig. 1 all the images relevant to the homogeneous test case are shown: the parameters of the ERS sensor have been used for the simulation and a subset of 256x256 pixels cropped from the simulated image is considered for the experiments. Values of all the quality measures for the case of interest are reported in Table I.

First of all, the presence of significant bias on the mean image is investigated, which is useful to gain insight into the behavior of the filters in terms of radiometric preservation. In fact, the mean value of the image (MoI) should be preserved through filtering. With this respect, we note that none of the considered filters introduces a significant bias on the mean. On the contrary, the mean value of the ratio image (MoR), obtained as pointwise ratio between original and filtered version of the same image, shows that Frost filter, unlike the other techniques, tends to "follow" the speckle, suggesting its imperfect removal.

In presence of a reference image, however, the measure of choice for noise rejection is the MSE, with the statistical average replaced here by sample average,

$\operatorname{MSE}(x, \hat{x})=\frac{1}{\operatorname{size}(x)} \sum_{i, j}(x(i, j)-\hat{x}(i, j))^{2}$,

where $\hat{x}$ is the filtered image and $x$ the 512-look reference. To improve readability of results, we will however show a related quantity, the despeckling gain defined as

$\mathrm{DG}=10 \log _{10}(\operatorname{MSE}(x, z) / \operatorname{MSE}(x, \hat{x}))$ 
TABLE II

\begin{tabular}{ccccr}
\multicolumn{5}{c}{ MEASURES FOR DEM } \\
\hline \hline Clean & 1.000 & 0.984 & 2.40 \\
Noisy & 0.987 & & 3.55 & 0 \\
Frost & 0.978 & 0.810 & 2.97 & 1.70 \\
SAWBMMAE & 0.772 & 0.911 & 2.14 & 4.67 \\
SAR-BM3D & 0.953 & 0.833 & 2.45 & 5.19 \\
\hline \hline
\end{tabular}

where $z$ is the unfiltered image. Therefore, larger numbers indicate better speckle rejection. Together with the MSE we also report the ENL, for several good reasons: 1) it is the measure people are most used to in this field, providing immediate insight about speckle reduction ability; 2) it has a compelling physical meaning as the number of looks needed to reach the same speckle suppression level guaranteed by filtering; 3) it can be computed also in the absence of a reference image, provided a flat region can be identified, which allows comparisons beyond the present framework. On the down side, ENL depends on the presence and detectability of a relatively large flat region of the image. Numerical results, in terms of both DG and ENL, show that SAR-BM3D guarantees the best performance, followed by SAWBMMAE and eventually by Frost.

\subsection{DEM}

In this case a synthetic canonical DEM is provided as input to the simulator. In Fig. 2 zooms (128x128 pixels) of the test, reference and filtered images are presented: again the parameters of the ERS sensor have been used and a subset of $512 \times 512$ pixels is considered for the experiments. Values of all the quality measures for the case of interest are reported in Table II.

In this case, the MoI indicates a non-negligible bias for SAWBBMAE, while the MoR provides more controversial indications. Here, the preferential performance measure is the MSE or, equivalently, the DG. The best performance is given by SAR-BM3D, just above $5 \mathrm{~dB}$ (compare it with the $20 \mathrm{~dB}$ of the homogeneous case) with SAWBBMAE just under $5 \mathrm{~dB}$. Frost provides lower values, close to $2 \mathrm{~dB}$ gain.

Up to this moment, we have considered only speckle suppression measures. From now on, instead, we will consider feature preservation measures, which are equally important and must be balanced against the former. A significant synthetic indicator, which tries to provide a measure of texture preservation, is the coefficient of variation, $C_{x}=\sigma_{x} / \mu_{x}$, which accounts for the region heterogeneity. Good texture preservation can be hoped for only if this indicator, computed on the despeckled image, is close to the value expected for the original image [3]. A critical point of this measure is the selection of an area of constant statistical behavior, so as to actually measure only intra-texture and not also inter-texture variability. In terms
TABLE III

\begin{tabular}{ccc}
\multicolumn{3}{c}{ MEASURES FOR CORNER } \\
\hline \hline & $C_{\mathrm{NN}}$ & $C_{\mathrm{BG}}$ \\
\hline Clean & 7.18 & 30.54 \\
Noisy & 7.19 & 30.52 \\
Frost & 7.19 & 30.52 \\
SAWBMMAE & 1.81 & 16.30 \\
SAR-BM3D & 6.83 & 29.55 \\
\hline \hline
\end{tabular}

of coefficient of variation, SAR-BM3D is again the best, with a value of 2.45 , very close to the 2.40 of the 512-look image. Further important information can be obtained by studying the autocorrelation functions of the images, which, for lack of space, are not reported in the present paper.

\subsection{Corner}

In Fig. 3 a zoom (128x128 pixels) of all the images relevant to the corner test case are shown: also in this case the ERS sensor parameters are assumed in the simulations and a subset of $256 \times 256$ pixels is considered for the experiments.

In this case we want mainly to assess the radiometric preservation through the filtering process. To this end, we use two amplitude contrast measures. Let $x_{\mathrm{CF}}$ be the intensity observed in the corner reflector site, $x_{\mathrm{NN}}$ the average intensity in the surrounding region formed by the 8connected nearest neighbors and $x_{\mathrm{BG}}$ the average intensity of the background. Then we define,

$C_{\mathrm{NN}}=10 \log _{10} \frac{x_{\mathrm{CF}}}{x_{\mathrm{NN}}}$

$C_{\mathrm{BG}}=10 \log _{10} \frac{x_{\mathrm{CF}}}{x_{\mathrm{BG}}}$.

In the filtered images, these figures should be as close as possible to the corresponding numbers computed on the reference image.

The values of $C_{\mathrm{NN}}$ and $C_{\mathrm{BG}}$ for the case of interest are reported in Table III. In this case the best performance is obtained by Frost, which preserve well the shape of the point target response, as testified by both the values reported in Tab. III. Conversely, SAWBMMAE strongly affects the radiometric properties of the corner signature, giving rise to a very strong smoothing effect.

\section{DISCUSSION}

In this paper we described a new approach to the assessment of SAR despeckling filters, and reported some experimental results for just a few scenes, measures and filters (more results are reported in [7]). This small sample, however, is enough to gain insight into the value of this approach. The proposed approach leads to fully reproducible experiments with objective numerical results, allowing for the objective 


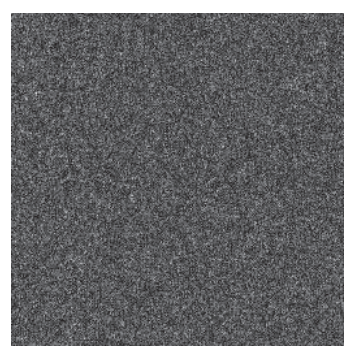

(a) SAR image

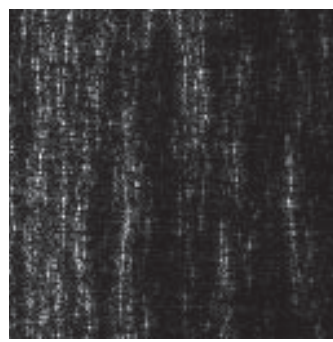

(a) SAR image

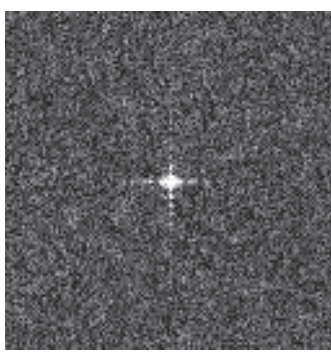

(a) SAR image

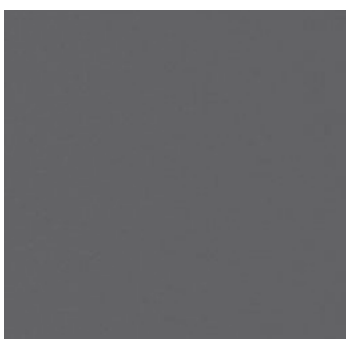

(b) Clean

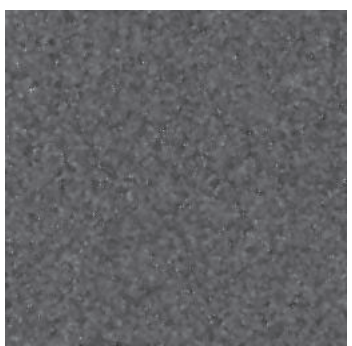

(c) Frost

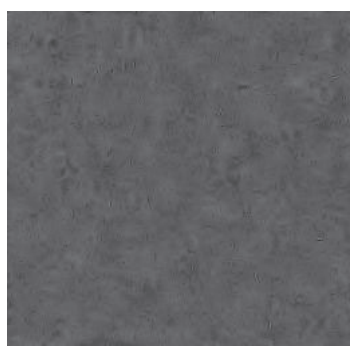

(d) SAWBMMAE

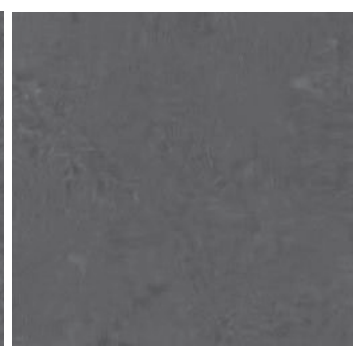

(e) SAR-BM3D

Fig. 1 Homogeneous: test, reference and output of the three filters.

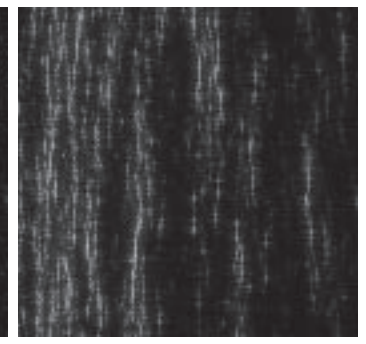

(b) Clean

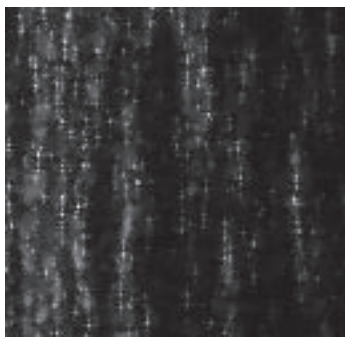

(c) Frost

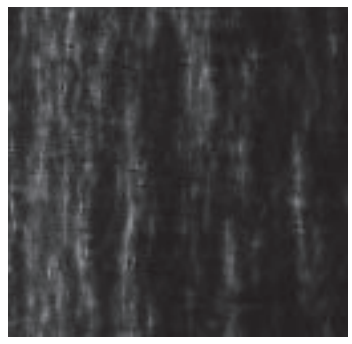

(d) SAWBMMAE

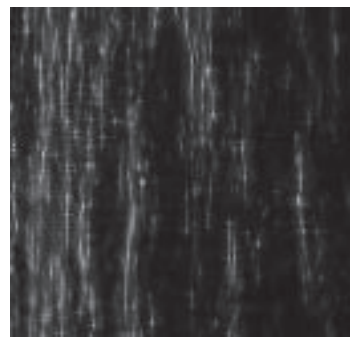

(e) SAR-BM3D

Fig. 2 DEM: test, reference and output of the three filters.

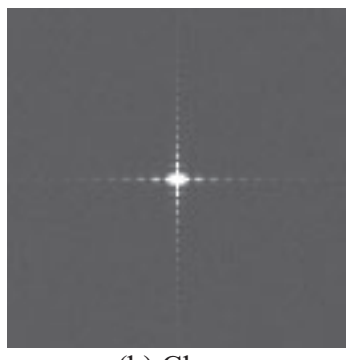

(b) Clean

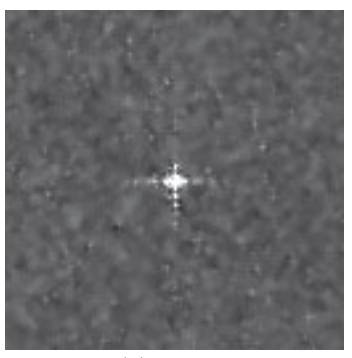

(c) Frost

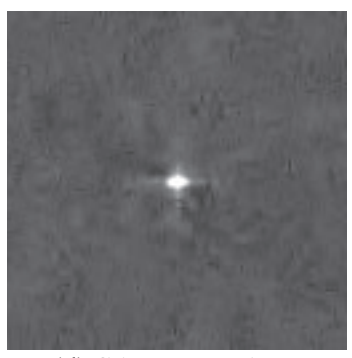

(d) SAWBMMAE

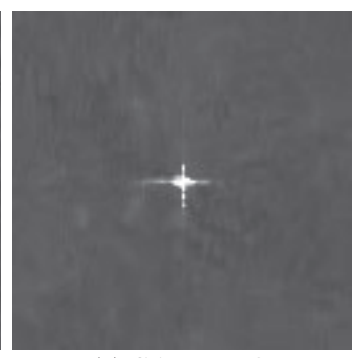

(e) SAR-BM3D

Fig. 3 Corner reflector: test, reference and output of the three filters.

comparison of competing techniques for any application of interest, as well as the fine tuning of new filters under test. In all our experiments, numerical results give indications that fully agree with the opinion of expert photo-interpreter, providing a sound support to this approach. More canonical scenes can then be selected, with specific performance measure, to enrich the test-bed an enlarge its scope.

\section{REFERENCES}

[1] M. Datcu, K. Seidel, and M. Walessa, "Spatial information retrieval from remote-sensing images. I. Information theoretical perspective," IEEE Trans. Geosci. Remote Sens., vol. 36, no. 5, pp. 1431-1445, Sep. 1998.

[2] J. W. Goodman, "Some fundamental properties of speckle", $J$. Opt. Soc. Am., vol. 66, no. 11, pp. 806-814, Nov. 1976.

[3] R. Touzi, "A review of speckle filtering in the context of estimation theory", IEEE Trans. Geosci. Remote Sens., vol. 40, no. 11, pp. 2392-2404, Nov. 2002.

[4] R. K. Raney and G. J. Wessels, "Spatial considerations in SAR speckle simulation", IEEE Trans. Geosci. Remote Sens., vol. 26, no. 5, pp. 666-672, Sep. 1988.
[5] G. Franceschetti, M. Migliaccio, D. Riccio, and G. Schirinzi, "SARAS: a SAR raw signal simulator", IEEE Trans. Geosci. Remote Sens., vol. 30, no. 1, pp. 110-123, Jan. 1992.

[6] G. Franceschetti and D. Riccio, Scattering, Natural Surfaces and Fractals. Burlington, MA: Academic, 2007.

[7] G. Di Martino, M. Poderico, G. Poggi, D. Riccio and L. Verdoliva, "Benchmarking framework for SAR despeckling", IEEE Trans. on Geosci. Remote Sens., submitted, 2012.

[8] V. S. Frost, J. A. Stiles, K. S. Shanmugan, and J. C. Holtzmann, "A model for radar images and its application to adaptive digital filtering of multiplicative noise", IEEE Trans. Pattern Anal. Machine Intell., vol. PAMI-4, no. 2, March 1982.

[9] M. I. H. Bhuiyan, M. O. Ahmad, and M. N. S. Swamy, "Spatially adaptive wavelet-based method using the Cauchy prior for denoising the SAR images", IEEE Trans. Circuits Syst. Video Technol., vol. 17, no. 4, pp. 500-507, April 2007.

[10] S. Parrilli, M. Poderico, C. V. Angelino, and L. Verdoliva, “A nonlocal SAR image denoising algorithm based on LLMMSE wavelet shrinkage," IEEE Trans. Geosci. Remote Sens., vol. 50, no. 2, pp. 606-616, Feb. 2012. 